\title{
Correction to: Cellular Reprogramming and Aging
}

\section{Correction to:}

G. Rodrigues, B. A. J. Roelen (eds.), Concepts and Applications of Stem Cell Biology, Learning Materials in Biosciences, https://doi.org/10.1007/978-3-030-43939-2_5

This chapter was inadvertently published with incorrect affiliation of second author Bruno Bernardes de Jesus which has been corrected as below:

The affiliation of Bruno Bernardes de Jesus has been changed to "Department of Medical Sciences and Institute of Biomedicine - iBiMED, University of Aveiro, Aveiro, Portugal". 